\title{
Persistent pemphigus vulgaris and pemphigus foliaceus showing features of tufted hair folliculitis just on the scalp
}

\author{
Maral Nouriyani', Fatemeh Montazer ${ }^{2}$, Fatemeh Rouzbeh ${ }^{3}$, Ramina Mofarrah ${ }^{4}$, \\ Ramin Mofarrah ${ }^{5}$
}

${ }^{1}$ Student Research Committee, Sari Branch, Islamic Azad University of Medical Sciences, Sari, Iran, ${ }^{2}$ Department of Pathology, Gastrointestinal Cancer Research Center, Imam Hospital, Mazandaran university of Medical Sciences, Sari, Iran, ${ }^{3}$ Department of Infectious Diseases, Sari Branch, Islamic Azad University, Sari, Iran, ${ }^{4}$ Student Research Committee, Shiraz Branch, University of Medical Sciences, Shiraz, Iran, ${ }^{5}$ Department of Dermatology, Faculty of Medicine, Islamic Azad University, Sari, Iran

Corresponding author: Ramin Mofarrah MD, E-mail: ramin.mofarrah@gmail.com

\begin{abstract}
Pemphigus vulgaris (PV) and pemphigus foliaceus $(\mathrm{PF})$ are rare autoimmune blistering diseases. The combination of these diseases together is very rare on the scalp. We report a 52-year-old female patient with localized painful crusted and prurient erosions and hair loss just on the scalp and no mucosal involvement. Scalp lesions had persisted about 4 years and the patient was wrongly diagnosed with psoriasis in these years. Clinical findings, as well as the results of biopsy and direct immunofluorescence, detected pemphigus vulgaris and pemphigus foliaceus also we have seen features of tufted hair folliculitis. treatment with systemic and topical corticosteroids resulted in clinical remission, with regrowth of scalp hair.
\end{abstract}

Key words: Pemphigus vulgaris; Pemphigus foliaceus; Tufted hair folliculitis; Scalp

\section{INTRODUCTION}

Pemphigus vulgaris and pemphigus foliaceus are rare diseases and the incidence of pemphigus depending on the area is 2 to 10 cases per one million [1]. According to our research no one has reported any cases in which these two types have occurred together on the scalp.

Pemphigus is a potentially life threatening autoimmune disease [2]. Two major types of pemphigus are pemphigus vulgaris and pemphigus foliaceus [3]. All pemphigus vulgaris patients have mucus involvement and more than half of them have skin involvement, but hair loss is rarely seen [3-5]. Oral lesions mostly appear in the buccal, palatine and gingival mucosa [6]. They appear a few weeks or months before skin lesions [7].

In pemphigus foliaceus, lesions only appear on the skin and there is rarely any mucus involvement $[3,6]$.
And these lesions often appear on the face and upper trunk. Compared to the pemphigus vulgaris, the blisters have a more superficial position in the epidermis [7]. In pemphigus disease, the immune system targets the desmoglein protein group and the intercellular transdermal desmosome disappear [6,7]. In pemphigus vulgaris it targets desmoglein 3 and in pemphigus fuliaceae it targets desmoglein 1 [5].

We report a rare case of pemphigus vulgaris and pemhigus foliaceus involving just the scalp that shows features of tufted hair folliculitis.

\section{CASE REPORT}

We report on a diabetic 52-year-old female patient with about a 4-year history of localized painful crusted and prurient erosions and these lesions did not leave

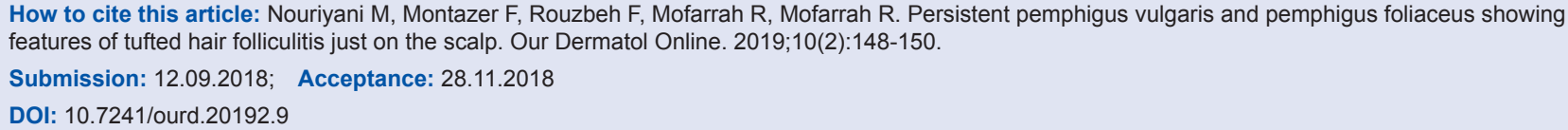


the scar tissue after recovery and there was hair loss only in those areas and she doesn't have any mucousal involvement (Fig. 1).

Apparent features of tufted hair folliculitis resulted in her illness being wrongly diagnosed as psoriasis and she used Daivobet gel and Clotrimazole for a while but she had frequent periods of remission and recurrence.

Her illness recurred during stress and in bad mental conditions and her erosions were persistent.

The patient also had a history of diabetes and she takes Metformin.

Biopsy was taken from the lesion in two samples from two separate parts and the histopathology findings were compatible with the diagnosis of pemphigus vulgaris (Fig. 2) and pemphigus foliaceus (Fig. 3),

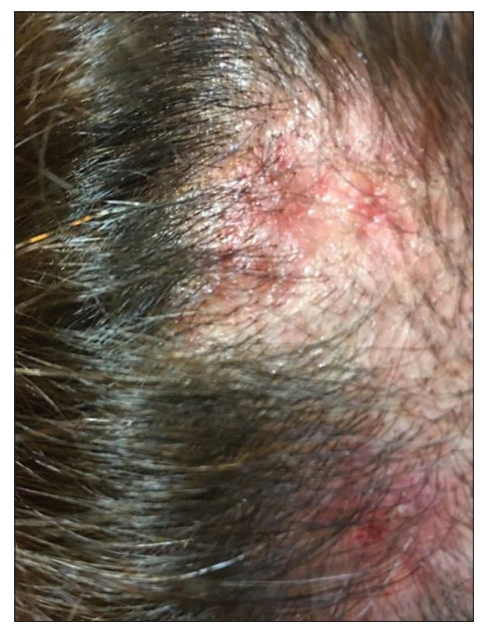

Figure 1: Scalp involvement in pemphigus vulgaris and pemphigus foliaceus.

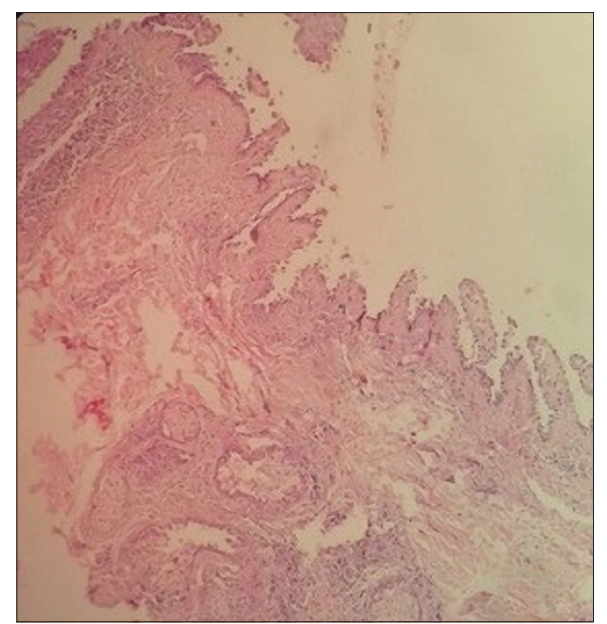

Figure 2: Pemphigus vulgaris: showing acantholysis and conspicuous villi $(\times 400)$. demonstrating acantholysis of the subcorneal and suprabasal area of entire epidermis.

Direct immunofluorescence demonstrated immunoglobulin G and Anti C3 deposition on the intercellular spaces within the epidermis (Fig. 4).

After rejecting the diagnosis of psoriasis and making the new diagnosis of pemphigus vulgaris and pemphigus foliaceus disease, the previous medications were discontinued.

The patient was treated with $7.5 \mathrm{mg}$ of Prednisolone for 3 weeks and after that the pill was tapered to a quarter per day for the rest of the 2-month treatment period. After that the patient finally discontinued oral treatment.

Also during this time, the patient used the topical combined treatment of Tacrolimus and Clobetasol $1 \%$.

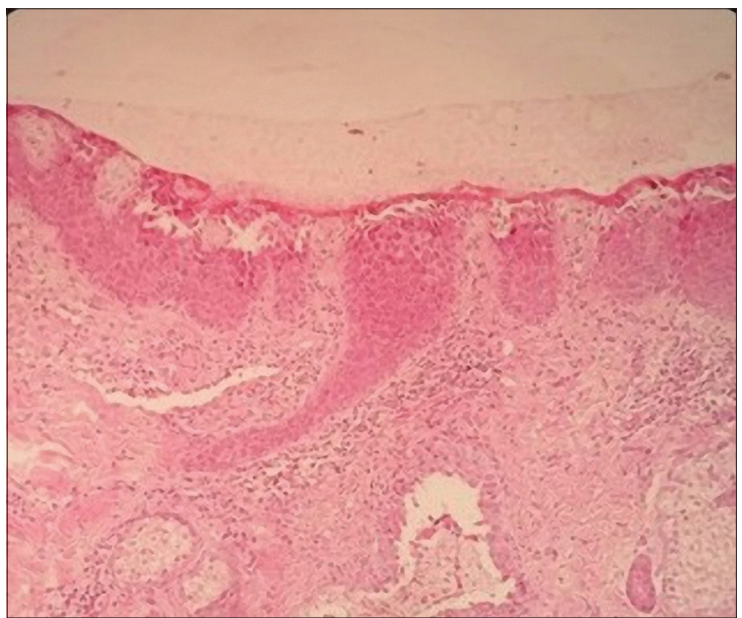

Figure 3: Pemphigus foliaceus: superficial acantholysis of epidermis within granular layer $(\times 100)$.

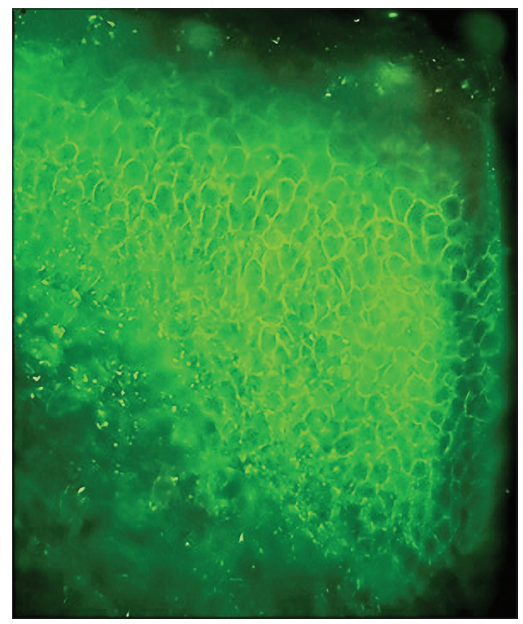

Figure 4: Direct immunofluorescence: typical intercellular staining (IgG) of the epidermis. 


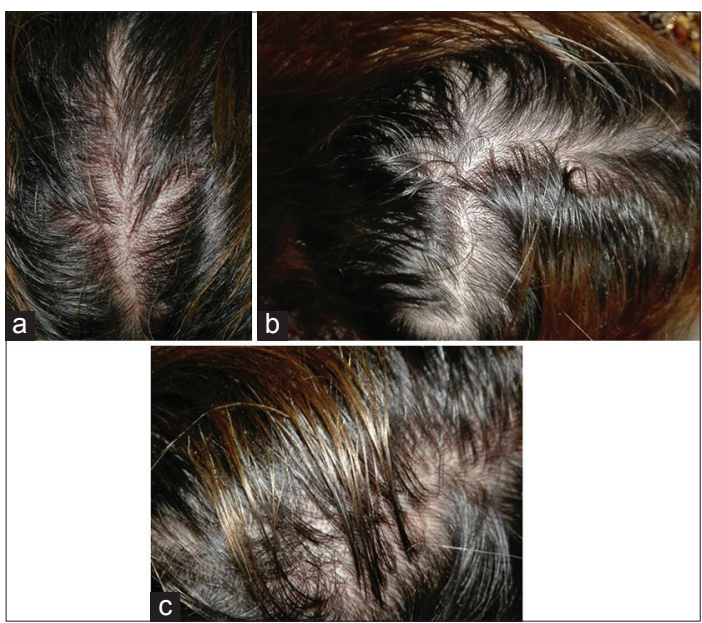

Figure 5: There is no lesions after treatment.

Eventually the patient discontinued the treatment after 3 months due to the rise of blood pressure and during the patient's follow up about 4 months later, her lesions had remissioned without relapse and regrowth of scalp hair could be seen (Figs. 5a-5c).

Prior to the study, patient gave written consent to the examination and biopsy after having been informed about the procedure.

\section{DISCUSSION}

Pemphigus vulgaris and pemphigus foliaceus are two major types of the rare disease pemphigus which cause skin lesions. They are characterized by the production of autoantibodies against intercellular substances and they are classified as autoimmune diseases.

The lesions were clinically similar to psoriasis and did not have the clinical appearance of pemphigus so we didn't consider it.

After taking a biopsy, psoriasis was rejected and pemphigus vulgaris and pemphigus foliaceus were confirmed so our final diagnosis was different from the initial diagnosis. Differential diagnoses of scalp involvement include psoriasis, seborrheic dermatitis, sebopsoriasis and cicatricial pemphigoid.

The cause of pemphigus vulgaris and its trigger is unknown and it is present in the scalp, but hair loss in this disease is rarely seen and is often caused by secondary infection $[4,5,9]$ most researchers report a secondary bacterial infection as the cause of hair folliculitis [5].
Apart from their rare scalp involvement, pemphigus vulgaris mostly involves the mucus and the skin while pemphigus foliaceus involves the skin. But none of them involve just the scalp.

In pemphigus foliaceus, cause of the disease is multifactorial and its pathogenesis is unknown and we cannot prevent the disease, but the impact of the environment and genetics has been proven [8] and preventing it is like pemphigus foliaceus.

Also pemphigus vulgaris in the early stages and pemphigus foliaceus in the final stages can have the same histopathology and DIF appearance.

The reason for the introduction of the case is due to the presence of both pemphigus simultaneously on the scalp, which has not yet been reported.

\section{Consent}

The examination of the patient was conducted according to the Declaration of Helsinki principles.

\section{REFERENCES}

1. DiMarco C. Pemphigus: Pathogenesis to Treatment. Rhode Island Med J. 2016;99:28-31.

2. Sar-Pomian M, Kurzeja M, Rudnicka L, Olszewska M. The value of trichoscopy in the differential diagnosis of scalp lesions in pemphigus vulgaris and pemphigus foliaceus. An Bras Dermatol. 2014;89:1007-12.

3. Amagai M. Pemphigus. In: Bolognia JL, Jorizzo JL, Schaffer JV, editors. Dermatology, $3^{\text {rd }}$ ed. London: Elsevier; 2012: 461-474.

4. Hadayer N, Ramot Y, Maly A, Zlotogorski A. Pemphigus vulgaris with loss of hair on the scalp. Int J Trichology. 2013;5:157-8.

5. Ko DK, Chae IS, Chung KH, Park JS, Chung H. Persistent pemphigus vulgaris showing features of tufted hair folliculitis. Ann Dermatol. 2011;23:523-5.

6. Payne AS, Stanley JR. Pemphigus. In: Goldsmith LA, Katz SI, Gilchrest BA, Paller AS, Leffell DJ, Wolff K, editors. Fitzpatrick's dermatology in general medicine, $8^{\text {th }}$ ed. New York: McGraw-Hill; 2007: 586-599.

7. High WA. Blistering diseases. In: Elston DM, Ferringer T, Ko CJ, Peckham S, High WA, DiCaudo DJ. Dermatopathology, $2^{\text {nd }}$ ed. London: Elsevier; 2014: 161-171.

8. Malheiros D, Panepucci RA, Roselino AM, Araújo AG, Zago pMA, Petzl-Erler ML. Genome-wide gene expression profiling reveals unsuspected molecular alterations in pemphigus foliaceus. Immunology. 2014;143:381-95.

9. Pirmez R. Acantholytic Hair Casts: A Dermoscopic Sign of Pemphigus Vulgaris of the Scalp. Int J Trichology. 2012;4:172-3.

Copyright by Maral Nouriyani, et al. This is an open-access article distributed under the terms of the Creative Commons Attribution License, which permits unrestricted use, distribution, and reproduction in any medium, provided the original author and source are credited.

Source of Support: Nil, Conflict of Interest: None declared. 